\title{
Weak harmonic Maass forms of weight $5 / 2$ and a mock modular form for the partition function
}

\author{
Scott Ahlgren* and Nickolas Andersen
}

*Correspondence:

sahlgren@illinois.edu

Department of Mathematics,

University of Illinois, Urbana IL, USA

\section{Abstract}

We construct a natural basis for the space of weak harmonic Maass forms of weight $5 / 2$ on the full modular group. The nonholomorphic part of the first element of this basis encodes the values of the ordinary partition function $p(n)$. We obtain a formula for the coefficients of the mock modular forms of weight $5 / 2$ in terms of regularized inner products of weakly holomorphic modular forms of weight $-1 / 2$, and we obtain Hecke-type relations among these mock modular forms.

\section{Introduction}

A number of recent works have considered bases for spaces of weak harmonic Maass forms of small weight. Borcherds [4] and Zagier [25] (in their study of infinite product expansions of modular forms, among many other topics) made use of the basis $\left\{f_{d}\right\}_{d>0}$ defined by $f_{-d}=q^{-d}+O(q)$ for the space of weakly holomorphic modular forms of weight $1 / 2$ in the Kohnen plus space of level 4. Duke, Imamoḡlu and Tóth [14] extended this to a basis $\left\{f_{d}\right\}_{d \in \mathbb{Z}}$ for the space of weak harmonic Maass forms of the same weight and level and interpreted the coefficients in terms of cycle integrals of the modular $j$-function. In subsequent work, [16] they constructed a similar basis in the case of weight 2 for the full modular group, and related the coefficients of these forms to regularized inner products of an infinite family of modular functions. To construct these bases requires various types of Maass-Poincare series. These have played a fundamental role in the theory of weak harmonic Maass forms (see for example $[6,10]$ among many other works).

Here, we will construct a natural basis for the space of weak harmonic Maass forms of weight $5 / 2$ on $\mathrm{SL}_{2}(\mathbb{Z})$ with a certain multiplier. To develop the necessary notation, let the Dedekind eta-function be defined by

$$
\eta(z):=q^{\frac{1}{24}} \prod_{n \geq 1}\left(1-q^{n}\right), \quad q:=e^{2 \pi i z}, \quad z=x+i y \in \mathbb{H},
$$

where $\mathbb{H}$ denotes the complex upper half-plane. We have the generating function

$$
\eta^{-1}(z)=\sum_{n \geq 0} p(n) q^{n-\frac{1}{24}},
$$

where $p(n)$ is the ordinary partition function. The transformation property

$$
\eta(\gamma z)=\varepsilon(\gamma)(c z+d)^{\frac{1}{2}} \eta(z), \quad \gamma=\left(\begin{array}{ll}
a & b \\
c & d
\end{array}\right) \in \mathrm{SL}_{2}(\mathbb{Z})
$$

(0) 2015 Ahlgren and Andersen. This is an Open Access article distributed under the terms of the Creative Commons Attribution License (http://creativecommons.org/licenses/by/4.0), which permits unrestricted use, distribution, and reproduction in any medium, provided the original work is properly credited. 
defines a multiplier system $\varepsilon$ of weight $1 / 2$ on $\mathrm{SL}_{2}(\mathbb{Z})$ which takes values in the 24 -th roots of unity. Let $M_{-\frac{1}{2}}^{!}(\bar{\varepsilon})$ denote the space of weakly holomorphic modular forms of weight $-1 / 2$ and multiplier $\bar{\varepsilon}$ on $\mathrm{SL}_{2}(\mathbb{Z})$. Then $\eta^{-1}(z)$ is the first element of a natural basis $\left\{g_{m}\right\}$ for this space. To construct the basis, we set $g_{1}:=\eta^{-1}$, and for each $m \equiv 1 \bmod 24$ we define $g_{m}:=g_{1} P(j)$, where $P(j)$ is a suitable monic polynomial in the Hauptmodul $j(z)$ such that $g_{m}=q^{-\frac{m}{24}}+O\left(q^{\frac{23}{24}}\right)$. We list the first few examples here:

$$
\begin{aligned}
g_{1} & =\eta^{-1} \\
& =q^{-\frac{1}{24}}+q^{\frac{23}{24}}+2 q^{\frac{47}{24}}+3 q^{\frac{71}{24}}+5 q^{\frac{95}{24}}+7 q^{\frac{119}{24}}+11 q^{\frac{143}{24}}+15 q^{\frac{167}{24}}+O\left(q^{\frac{191}{24}}\right), \\
g_{25} & =\eta^{-1}(j-745) \\
& =q^{-\frac{25}{24}}+196885 q^{\frac{23}{24}}+21690645 q^{\frac{47}{24}}+886187500 q^{\frac{71}{24}}+O\left(q^{\frac{95}{24}}\right), \\
g_{49} & =\eta^{-1}\left(j^{2}-1489 j+160511\right) \\
& =q^{-\frac{49}{24}}+42790636 q^{\frac{23}{24}}+40513206272 q^{\frac{47}{24}}+8543738297129 q^{\frac{71}{24}}+O\left(q^{\frac{95}{24}}\right) .
\end{aligned}
$$

Denote by $H_{\frac{5}{2}}^{!}(\varepsilon)$ the space of weak harmonic Maass forms of weight $5 / 2$ and multiplier $\varepsilon$ on $\mathrm{SL}_{2}(\mathbb{Z})$. These are real analytic functions which transform as

$$
F(\gamma z)=\varepsilon(\gamma)(c z+d)^{\frac{5}{2}} F(z), \quad \gamma=\left(\begin{array}{ll}
a & b \\
c & d
\end{array}\right) \in \mathrm{SL}_{2}(\mathbb{Z}),
$$

which are annihilated by the weight $5 / 2$ hyperbolic Laplacian

$$
\Delta_{\frac{5}{2}}:=-y^{2}\left(\frac{\partial^{2}}{\partial x^{2}}+\frac{\partial^{2}}{\partial y^{2}}\right)+\frac{5}{2} i y\left(\frac{\partial}{\partial x}+i \frac{\partial}{\partial y}\right)
$$

and which have at most linear exponential growth at $\infty$ (see the next section for details). If $M_{\frac{5}{2}}^{!}(\varepsilon)$ denotes the space of weakly holomorphic modular forms of weight $5 / 2$ and multiplier $\varepsilon$, then we have $M_{\frac{5}{2}}^{!}(\varepsilon) \subseteq H_{\frac{5}{2}}^{!}(\varepsilon)$.

Here we construct a basis $\left\{h_{m}\right\}$ for the space $H_{\frac{5}{2}}^{!}(\varepsilon)$. For negative $m$ we have $h_{m} \in$ $M_{\frac{5}{2}}^{!}(\varepsilon)$, while for positive $m$ we have $\xi_{\frac{5}{2}}\left(h_{m}\right)=\left(\frac{6}{\pi m}\right)^{\frac{3}{2}} g_{m}$, where $\xi_{\frac{5}{2}}: H_{\frac{5}{2}}^{!}(\varepsilon) \rightarrow M_{-\frac{1}{2}}^{!}(\bar{\varepsilon})$ is the $\xi$-operator defined in (2.2) below. In particular, the nonholomorphic part of $h_{1}$ encodes the values of the partition function.

To state the first result it will be convenient to introduce the notation

$$
\beta(y)=\Gamma\left(-\frac{3}{2}, \frac{\pi y}{6}\right),
$$

where the incomplete gamma function is given by $\Gamma(s, y):=\int_{y}^{\infty} e^{-t} t^{s-1} d t$.

Theorem 1. For each $m \equiv 1(\bmod 24)$, there is a unique $h_{m} \in H_{\frac{5}{2}}^{!}(\varepsilon)$ with Fourier expansion of the form

$$
h_{m}(z)=q^{\frac{m}{24}}+\sum_{0<n \equiv 1(24)} p_{m}^{+}(n) q^{\frac{n}{24}} \quad \text { if } m<0,
$$

and

$$
h_{m}(z)=\sum_{0<n \equiv 1(24)} p_{m}^{+}(n) q^{\frac{n}{24}}+i \beta(-m y) q^{\frac{m}{24}}+\sum_{0<n \equiv-1(24)} p_{m}^{-}(n) \beta(n y) q^{-\frac{n}{24}} \quad \text { if } m>0 .
$$


The set $\left\{h_{m}\right\}$ forms a basis for $H_{\frac{5}{2}}^{!}(\varepsilon)$. For $m<0$ we have $h_{m} \in M_{\frac{5}{2}}^{!}(\varepsilon)$, while for $m>0$ we have

$$
\xi_{\frac{5}{2}}\left(h_{m}\right)=\left(\frac{6}{\pi m}\right)^{\frac{3}{2}} \cdot g_{m} \in M_{-\frac{1}{2}}^{!}(\bar{\varepsilon}) .
$$

Furthermore, for $m \neq n$, the coefficients $p_{m}^{+}(n)$ are real.

When $m=1$, we have

$$
\xi_{\frac{5}{2}}\left(h_{1}\right)=\left(\frac{6}{\pi}\right)^{\frac{3}{2}} \eta^{-1}(z)=\left(\frac{6}{\pi}\right)^{\frac{3}{2}} \sum_{n \geq-1} p\left(\frac{n+1}{24}\right) q^{\frac{n}{24}}
$$

Using Lemma 7 below we obtain the following corollary.

Corollary 2. The function $\mathbf{P}(z)$ defined by

$$
\boldsymbol{P}(z):=\sum_{0<n \equiv 1(24)} p_{1}^{+}(n) q^{\frac{n}{24}}-\sum_{-1 \leq n \equiv 23(24)} p\left(\frac{n+1}{24}\right) n^{\frac{3}{2}} \beta(n y) q^{-\frac{n}{24}}
$$

is a weight $\frac{5}{2}$ weak harmonic Maass form on $S L_{2}(\mathbb{Z})$ with multiplier $\varepsilon$.

The coefficients can be computed using the formula in Proposition 11 below. We have

$$
\begin{array}{r}
\mathbf{P}(z)=(-3.96 \ldots+ \\
\left.+i \frac{4}{3} \sqrt{\pi}\right) q^{\frac{1}{24}}+111.40 \ldots q^{\frac{25}{24}}+254.26 \ldots q^{\frac{49}{24}}+86.52 \ldots q^{\frac{73}{24}}+\cdots \\
+i \beta(-y) q^{\frac{1}{24}}-1 \cdot 23^{\frac{3}{2}} \beta(23 y) q^{-\frac{23}{24}}-2 \cdot 47^{\frac{3}{2}} \beta(47 y) q^{-\frac{47}{24}}+\cdots
\end{array}
$$

We will construct the functions $h_{m}(z)$ in Section 3 using Maass-Poincaré series. Unfortunately, the standard construction does not produce any nonholomorphic forms in $H_{\frac{5}{2}}^{!}(\varepsilon)$, and we must therefore consider derivatives of these series with respect to an auxiliary parameter. This method was recently used by Duke, Imamoḡlu and Tóth [16] in the case of weight 2 (see also $[5,20]$ ). The construction provides an exact formula for the coefficients $p_{m}^{ \pm}(n)$. In particular, when $m=1$ we obtain the famous exact formula of Rademacher [23] for $p(n)$ as a corollary (see Section 4 for details).

Work of Bruinier and Ono [11] provides an algebraic formula for the coefficients $p_{m}^{-}(n)$ (and in particular the values of $p(n)$ ) as the trace of certain weak Maass forms over CM points. Forthcoming work of the second author [3] investigates the analogous arithmetic and geometric nature of the coefficients $p_{m}^{+}(n)$. In analogy with [14] and [9], the coefficients are interpreted as the real quadratic traces (i.e. sums of cycle integrals) of weak Maass forms. 
Remark. When $m<0$, we can construct the forms $h_{m}$ directly as in (1.2). We list a few examples here. Let $j^{\prime}:=-q \frac{d}{d q} j$. Then

$$
\begin{aligned}
h_{-23} & =\eta j^{\prime} \\
& =q^{-\frac{23}{24}}-q^{\frac{1}{24}}-196885 q^{\frac{25}{24}}-42790636 q^{\frac{49}{24}}-2549715506 q^{\frac{73}{24}}+O\left(q^{\frac{97}{24}}\right), \\
h_{-47} & =h_{-23}(j-743) \\
& =q^{-\frac{47}{24}}-2 q^{\frac{1}{24}}-21690645 q^{\frac{25}{24}}-40513206272 q^{\frac{49}{24}}+O\left(q^{\frac{73}{24}}\right), \\
h_{-71} & =h_{-23}\left(j^{2}-1487 j+355910\right) \\
& =q^{-\frac{71}{24}}-3 q^{\frac{1}{24}}-886187500 q^{\frac{25}{24}}-8543738297129 q^{\frac{49}{24}}+O\left(q^{\frac{73}{24}}\right) .
\end{aligned}
$$

Together with the family (1.2), these form a "grid" in the sense of Guerzhoy [17] or Zagier [25] (note that the integers appearing as coefficients are the same up to sign as those in $(1.2))$.

For $m>0$, the formula for $p_{m}^{+}(n)$ which results from the construction is an infinite series whose terms are Kloosterman sums multiplied by a derivative of the $J$-Bessel function in its index. Here we give an alternate interpretation of these coefficients involving the regularized Petersson inner product, in analogy with [16] and [15].

For modular forms $f$ and $g$ of weight $k$, define

$$
\langle f, g\rangle_{\mathrm{reg}}=\lim _{Y \rightarrow \infty} \int_{\mathcal{F}(Y)} f(z) \overline{g(z)} y^{k} \frac{d x d y}{y^{2}},
$$

provided that this limit exists. Here $\mathcal{F}(Y)$ denotes the usual fundamental domain for $\mathrm{SL}_{2}(\mathbb{Z})$ truncated at height $Y$.

Theorem 3. For positive $m, n \equiv 1(\bmod 24)$ with $m \neq n$ we have

$$
p_{m}^{+}(n):=\left(\frac{6}{\pi m}\right)^{\frac{3}{2}} \cdot\left\langle g_{m}, g_{n}\right\rangle_{\mathrm{reg}} .
$$

We note that when $n=m$ the integral defining this inner product does not converge.

The following is an immediate corollary of Theorem 3.

Corollary 4. For positive $m, n \equiv 1(\bmod 24)$ we have

$$
n^{\frac{3}{2}} \cdot p_{n}^{+}(m)=m^{\frac{3}{2}} \cdot p_{m}^{+}(n) \text {. }
$$

There are also Hecke relations among the forms $h_{m}$. Let $T_{\frac{5}{2}}\left(\ell^{2}\right)$ denote the Hecke operator of index $\ell^{2}$ on $H_{\frac{5}{2}}^{!}(\varepsilon)$ (see Section 6 for definitions).

Theorem 5. For any $m \equiv 1(\bmod 24)$ and for any prime $\ell \geq 5$ we have

$$
h_{m} \mid T_{\frac{5}{2}}\left(\ell^{2}\right)=\ell^{3} h_{\ell^{2} m}+\left(\frac{3 m}{\ell}\right) \ell h_{m} .
$$

Using Theorem 5 it is possible to deduce many relations among the coefficients $p_{m}^{+}(n)$. We record a typical example as a corollary. 
Corollary 6. If $m, n \equiv 1(\bmod 24)$ and $\ell \geq 5$ is a prime with $\left(\frac{m}{\ell}\right)=\left(\frac{n}{\ell}\right)$, then

$$
p_{m}^{+}\left(\ell^{2} n\right)=\ell^{3} p_{\ell^{2} m}^{+}(n) \text {. }
$$

Remark. It is possible to derive results analogous to Theorem 5 and Corollary 6 involving the operators $T_{\frac{5}{2}}\left(\ell^{2 k}\right)$ for any $k$ (see, for example, $\left.[1,2]\right)$. For brevity, we will not record these statements here.

In the next section we provide some background material. In Section 3 we adapt the method of [16] to construct the basis described in Theorem 1 . The last three sections contain the proofs of the remaining results.

\section{Weak harmonic Maass forms}

We require some preliminaries on weak harmonic Maass forms (see for example $[8,22$, 24] for further details). For convenience, we set $\Gamma:=\mathrm{SL}_{2}(\mathbb{Z})$. If $k \in \frac{1}{2} \mathbb{Z}$ then we say that $f$ has weight $k$ and multiplier $v$ for $\Gamma$ if

$$
\left.f\right|_{k} \gamma=v(\gamma) f
$$

for every $\gamma \in \Gamma$. Here $\left.\right|_{k}$ denotes the slash operator defined for $\gamma=\left(\begin{array}{ll}a & b \\ c & d\end{array}\right) \in \mathrm{GL}_{2}(\mathbb{Q})$ with $\operatorname{det}(\gamma)>0$ by

$$
\left.f\right|_{k} \gamma:=(\operatorname{det} \gamma)^{\frac{k}{2}}(c z+d)^{-k} f\left(\frac{a z+b}{c z+d}\right) .
$$

We choose the argument of each nonzero $\tau \in \mathbb{C}$ in $(-\pi, \pi]$, and we define $\tau^{k}$ using the principal branch of the logarithm. For any $k \in \frac{1}{2} \mathbb{Z}$, let $\xi_{k}$ denote the Maass-type differential operator which acts on differentiable functions $f$ on $\mathbb{H}$ by

$$
\xi_{k}(f)=2 i y^{k} \frac{\overline{\partial f}}{\partial \bar{z}}
$$

This operator satisfies

$$
\xi_{k}\left(\left.f\right|_{k} \gamma\right)=\left.\left(\xi_{k} f\right)\right|_{2-k} \gamma
$$

for any $\gamma \in \Gamma$. So if $f$ is modular of weight $k$, then $\xi_{k}(f)$ is modular of weight $2-k$. Furthermore, $\xi_{k}(f)=0$ if and only if $f$ is holomorphic. We define the weight $k$ hyperbolic Laplacian $\Delta_{k}$ by

$$
\Delta_{k}:=-\xi_{2-k} \circ \xi_{k}=-y^{2}\left(\frac{\partial^{2}}{\partial x^{2}}+\frac{\partial^{2}}{\partial y^{2}}\right)+i k y\left(\frac{\partial}{\partial x}+i \frac{\partial}{\partial y}\right) .
$$

In this paper, we are interested in the multiplier system $\varepsilon$ which is attached to the Dedekind eta function. An explicit description of $\varepsilon$ is given, for instance, in ([19], Section 2.8). Defining $e(\alpha):=e^{2 \pi i \alpha}$, we have $\varepsilon\left(\left(\begin{array}{ll}1 & 1 \\ 0 & 1\end{array}\right)\right)=e\left(\frac{1}{24}\right)$ and $\varepsilon(-I)=e\left(-\frac{1}{4}\right)$. For $\gamma=\left(\begin{array}{ll}a & b \\ c & d\end{array}\right)$ with $c>0$, we have

$$
\varepsilon(\gamma)=e\left(\frac{a+d-3 c}{24 c}-\frac{1}{2} s(d, c)\right),
$$


where $s(d, c)$ is the Dedekind sum

$$
s(d, c):=\sum_{r=1}^{c-1}\left(\frac{r}{c}-\frac{1}{2}\right)\left(\frac{d r}{c}-\left\lfloor\frac{d r}{c}\right\rfloor-\frac{1}{2}\right) .
$$

If $f \neq 0$ satisfies $(2.1)$ with $v=\varepsilon$ then we must have $2 k \equiv 1(\bmod 4)$, since

$$
(-1)^{-k} f=\left.f\right|_{k}(-I)=\varepsilon(-I) f .
$$

In what follows, we assume this consistency condition so that the forms in question are not identically zero.

Suppose that $f: \mathbb{H} \rightarrow \mathbb{C}$ is real analytic and satisfies

$$
\left.f\right|_{k} \gamma=\varepsilon(\gamma) f
$$

for all $\gamma \in \Gamma$. Then $f$ has a Fourier expansion at $\infty$ which is supported on exponents of the form $n / 24$ with $n \equiv 1(\bmod 24)$. If, in addition, $f$ satisfies

$$
\Delta_{k} f=0,
$$

then by the discussion in Section 3.2 below we have the Fourier expansion

$$
f(z)=\sum_{n \equiv 1(24)} a^{+}(n) q^{\frac{n}{24}}+\sum_{n \equiv-1(24)} a^{-}(n) \Gamma\left(1-k, \frac{\pi n y}{6}\right) q^{-\frac{n}{24}} .
$$

Let $H_{k}^{\prime}(\varepsilon)$ denote the space of functions satisfying (2.7) and (2.8) with the additional property that only finitely many of the $a^{ \pm}(n)$ with $n \leq 0$ in (2.9) are nonzero. We call elements of $H_{k}^{!}(\varepsilon)$ weak harmonic Maass forms of weight $k$ and multiplier $\varepsilon$. Note that these forms are allowed to have poles in the nonholomorphic part. Let $S_{k}(\varepsilon) \subseteq M_{k}(\varepsilon) \subseteq M_{k}^{!}(\varepsilon) \subseteq$ $H_{k}^{!}(\varepsilon)$ denote the subspaces of cusp forms, modular forms, and weakly holomorphic modular forms, respectively.

The next lemma follows from a computation. Care must be taken with the two cases $m>0$ and $m<0$.

Lemma 7. For any $m$ and any constant $c$, we have

$$
\xi_{\frac{5}{2}}\left(c \cdot \beta(m y) q^{-\frac{m}{24}}\right)=-\bar{c} \cdot\left(\frac{6}{\pi m}\right)^{\frac{3}{2}} q^{\frac{m}{24}} .
$$

Suppose that $f \in H_{\frac{5}{2}}^{!}(\varepsilon)$ has Fourier expansion (2.9). By Lemma 7 and (2.3) we find that

$$
\xi_{\frac{5}{2}}(f)=-\sum_{n \equiv-1(24)} \overline{a^{-}(n)}\left(\frac{6}{\pi n}\right)^{\frac{3}{2}} q^{\frac{n}{24}} \in M_{-\frac{1}{2}}^{!}(\bar{\varepsilon}) .
$$

Since $S_{-\frac{1}{2}}(\bar{\varepsilon})=\{0\}$ and $S_{\frac{5}{2}}(\varepsilon)=\{0\}$, we see that $f=0$ if and only if $a^{ \pm}(n)=0$ for all $n<0$. Because of this, each form $h_{m}$ in Theorem 1 is uniquely determined by a single term. If $m<0$ this term is $q^{\frac{m}{24}}$ and if $m>0$ this term is $\beta(-m y) q^{\frac{m}{24}}$.

\section{Construction of harmonic Maass forms and proof of Theorem 1}

In order to construct the basis described in Theorem 1, we first construct Poincaré series attached to the usual Whittaker functions (similar constructions can be found in [5, 7, $10,14,16,20$ ] among others). It turns out that for positive $m$ these series are identically zero. So we must differentiate with respect to an auxiliary parameter $s$ in order to obtain nontrivial forms in this case. The construction is carried out in several subsections and is summarized in Proposition 11 below. 


\subsection{Poincaré series}

In this section, fix $k \in \frac{1}{2} \mathbb{Z}$ with $2 k \equiv 1(\bmod 4)$. Suppose that $\varphi(z)$ is a smooth function satisfying $\varphi(z+1)=e\left(\frac{1}{24}\right) \varphi(z)$. Since $\varepsilon\left(\left(\begin{array}{cc}1 & n \\ 0 & 1\end{array}\right) \gamma\right)=e\left(\frac{n}{24}\right) \varepsilon(\gamma)$, the expression

$$
\bar{\varepsilon}(\gamma)(c z+d)^{-k} \varphi(\gamma z)
$$

only depends on the coset $\Gamma_{\infty} \gamma$, where $\Gamma_{\infty}:=\left\{\left(\begin{array}{ll}1 & n \\ 0 & 1\end{array}\right): n \in \mathbb{Z}\right\}$. So the series

$$
P(z):=\sum_{\gamma \in \Gamma_{\infty} \backslash \Gamma} \bar{\varepsilon}(\gamma)(c z+d)^{-k} \varphi(\gamma z)
$$

is well-defined if $\varphi$ is chosen so that it converges absolutely. Each coset of $\Gamma_{\infty} \backslash \Gamma$ corresponds to a pair $(c, d)$ with $c \in \mathbb{Z}$ and $(d, c)=1$. Because of (2.6) the terms corresponding to $c$ and $-c$ are equal.

Let $m \equiv 1(\bmod 24)$ and define

$$
\varphi_{m}(z):=\varphi^{0}\left(\frac{y}{24}\right) e\left(\frac{m x}{24}\right),
$$

with $\varphi^{0}(y)=O\left(y^{\alpha}\right)$ as $y \rightarrow 0$ for some $\alpha \in \mathbb{R}$.

As in the proof of the convergence of the classical Eisenstein series, we find that the series

$$
P_{m}(z):=\frac{1}{2} \sum_{\gamma \in \Gamma_{\infty} \backslash \Gamma} \bar{\varepsilon}(\gamma)(c z+d)^{-k} \varphi_{m}(\gamma z)
$$

converges absolutely and locally uniformly for $k>2-2 \alpha$.

The function $P_{m}(z)-\varphi_{m}(z)$ has polynomial growth as $y \rightarrow \infty$, and the function $e\left(-\frac{x}{24}\right)\left(P_{m}(z)-\varphi_{m}(z)\right)$ is periodic with period 1. So we have the Fourier expansion

$$
e\left(-\frac{x}{24}\right)\left(P_{m}(z)-\varphi_{m}(z)\right)=\sum_{n \in \mathbb{Z}} a(n, y) e(n x)
$$

with

$$
a(n, y)=\int_{0}^{1} e\left(-\frac{x}{24}\right)\left(P_{m}(z)-\varphi_{m}(z)\right) e(-n x) d x
$$

Using a standard argument (see, for example, [10], Proposition 3.1) we compute the Fourier coefficients $a(n, y)$ as

$$
\begin{aligned}
a(n, y)= & \int_{0}^{1} e\left(-\frac{x}{24}\right) \sum_{\substack{\gamma \in \Gamma_{\infty} \backslash \Gamma \\
c>0}} \bar{\varepsilon}(\gamma)(c z+d)^{-k} \varphi(\gamma z) e(-n x) d x \\
= & \sum_{\substack{(c, d)=1 \\
c>0}} \frac{\bar{\varepsilon}(\gamma)}{c^{k}} \int_{\frac{d}{c}}^{1+\frac{d}{c}} e\left(\frac{-x+d / c}{24}\right) z^{-k} \varphi\left(\frac{a}{c}-\frac{1}{c^{2} z}\right) e\left(-n x+\frac{n d}{c}\right) d x \\
= & \sum_{\substack{c>0 \\
d \bmod c^{*}}} \frac{\bar{\varepsilon}(\gamma)}{c^{k}} e\left(\frac{\bar{d} m+d(24 n+1)}{24 c}\right) \\
& \times \int_{-\infty}^{\infty} z^{-k} \varphi^{0}\left(\frac{y}{24 c^{2}|z|^{2}}\right) e\left(-\frac{m x}{24 c^{2}|z|^{2}}-\left(n+\frac{1}{24}\right) x\right) d x .
\end{aligned}
$$

Here $c^{*}$ indicates that the sum is restricted to residue classes coprime to $c$, and $\bar{d}$ denotes the inverse of $d$ modulo $c$. The second equality comes from writing $\gamma z=\frac{a}{c}-\frac{1}{c^{2}(z+d / c)}$ and from making the change of variable $x \rightarrow x-d / c$. The last equality comes from writing $d=d^{\prime}+\ell c$ with $0 \leq d^{\prime}<c$ and gluing together the integrals for each $\ell$. 
If we write $m=24 m^{\prime}+1$, then by (2.4) we obtain

$$
\sum_{d \bmod c^{*}} \bar{\varepsilon}(\gamma) e\left(\frac{\bar{d} m+d(24 n+1)}{24 c}\right)=\sqrt{i} K\left(m^{\prime}, n, c\right),
$$

where $K(m, n, c)$ denotes the Kloosterman sum

$$
K(m, n ; c):=\sum_{d \bmod c^{*}} e^{\pi i s(d, c)} e\left(\frac{\bar{d} m+d n}{c}\right) .
$$

Therefore we have

$$
P_{m}(z)=\varphi_{m}(z)+\sum_{n \in \mathbb{Z}} c(n, y) e\left(\left(n+\frac{1}{24}\right) x\right)
$$

with

$$
c(n, y)=\sqrt{i} \sum_{c>0} \frac{K\left(m^{\prime}, n ; c\right)}{c^{k}} \int_{-\infty}^{\infty} z^{-k} \varphi^{0}\left(\frac{y}{24 c^{2}|z|^{2}}\right) e\left(-\frac{m x}{24 c^{2}|z|^{2}}-\left(n+\frac{1}{24}\right) x\right) d x .
$$

\subsection{Whittaker functions and nonholomorphic Maass-Poincaré series}

The Poincaré series $P_{m}(z)$ clearly has the desired transformation behavior; in order to construct harmonic forms, we specialize $\varphi^{0}(y)$ to be a function which has the desired behavior under $\Delta_{k}$. The Whittaker functions $M_{\mu, v}(y)$ and $W_{\mu, v}(y)$ are linearly independent solutions of Whittaker's differential equation

$$
w^{\prime \prime}+\left(-\frac{1}{4}+\frac{\mu}{y}+\frac{1-4 v^{2}}{4 y^{2}}\right) w=0,
$$

and are defined in terms of confluent hypergeometric functions (see ([21], §13.14) for definitions and properties). Using (3.5) we see after a computation that

$$
y^{-\frac{k}{2}} M_{\operatorname{sgn}(n) \frac{k}{2}, s-\frac{1}{2}}(4 \pi|n| y) e(n x) \quad \text { and } \quad y^{-\frac{k}{2}} W_{\operatorname{sgn}(n) \frac{k}{2}, s-\frac{1}{2}}(4 \pi|n| y) e(n x)
$$

are linearly independent solutions of the differential equation

$$
\Delta_{k} F(z)=\left(s-\frac{k}{2}\right)\left(1-\frac{k}{2}-s\right) F(z) .
$$

At the special value $s=\frac{k}{2}$, we have (by [21], (13.18.2), (13.18.5))

$$
y^{-\frac{k}{2}} M_{\operatorname{sgn}(n) \frac{k}{2}, \frac{k-1}{2}}(y)=e^{-\operatorname{sgn}(n) \frac{y}{2}}
$$

and

$$
y^{-\frac{k}{2}} W_{\operatorname{sgn}(n) \frac{k}{2}, \frac{k-1}{2}}(y)= \begin{cases}e^{-\frac{y}{2}} & \text { if } n>0, \\ \Gamma(1-k, y) e^{\frac{y}{2}} & \text { if } n<0 .\end{cases}
$$

For $m \equiv 1(\bmod 24)$ and $s \in \mathbb{C}$, define

$$
\varphi_{m, s}(z):=\left(4 \pi|m| \frac{y}{24}\right)^{-\frac{k}{2}} M_{\operatorname{sgn}(m) \frac{k}{2}, s-\frac{1}{2}}\left(4 \pi|m| \frac{y}{24}\right) e\left(\frac{m x}{24}\right) .
$$

Then $\varphi_{m, s}(z)=O\left(y^{\operatorname{Re}(s)-\frac{k}{2}}\right)$ as $y \rightarrow 0$, so the series

$$
P_{m}(z, s):=\frac{1}{2} \sum_{\gamma \in \Gamma_{\infty} \backslash \Gamma} \bar{\varepsilon}(\gamma)(c z+d)^{-k} \varphi_{m, s}(\gamma z)
$$

converges for $\operatorname{Re}(s)>1$. Thus if one of the special values $s=\frac{k}{2}$ or $s=1-\frac{k}{2}$ is larger than 1 , then $P_{m}(z, s)$ is harmonic at this value. The following proposition describes the Fourier expansion of $P_{m}(z, s)$. 
Proposition 8. Let $k$ and $P_{m}(z, s)$ be as above, and suppose that $m \equiv 1(\bmod 24)$ and $\operatorname{Re}(s)>1$. Then we have

$$
P_{m}(z, s)=\varphi_{m, s}(z)+\sum_{n \equiv 1(24)} g_{m, n}(s) L_{m, n}(s)\left(4 \pi|m| \frac{y}{24}\right)^{-\frac{k}{2}} W_{\operatorname{sgn}(n) \frac{k}{2}, s-\frac{1}{2}}\left(4 \pi|n| \frac{y}{24}\right) e\left(\frac{n x}{24}\right)
$$

where

$$
g_{m, n}(s):=\frac{2 \pi i^{-k+\frac{1}{2}} \Gamma(2 s) \sqrt{|m / n|}}{\Gamma\left(s+\operatorname{sgn}(n) \frac{k}{2}\right)}
$$

and

$$
L_{m, n}(s):=\left\{\begin{array}{l}
\sum_{c>0} \frac{K\left(m^{\prime}, n^{\prime} ; c\right)}{c} I_{2 s-1}\left(\frac{\pi \sqrt{|m n|}}{6 c}\right) \text { if } m n>0, \\
\sum_{c>0} \frac{K\left(m^{\prime}, n^{\prime} ; c\right)}{c} I_{2 s-1}\left(\frac{\pi \sqrt{|m n|}}{6 c}\right) \text { if } m n<0 .
\end{array}\right.
$$

Here $J_{\alpha}(x)$ and $I_{\alpha}(x)$ denote the usual Bessel functions and $K\left(m^{\prime}, n^{\prime} ; c\right)$ is defined in (3.2).

Proof. In view of (3.9) and (3.1) we take

$$
\varphi^{0}(y):=(4 \pi|m| y)^{-\frac{k}{2}} M_{\operatorname{sgn}(m) \frac{k}{2}, s-\frac{1}{2}}(4 \pi|m| y) .
$$

We write

$$
n=24 n^{\prime}+1 .
$$

Then (3.3) becomes

$$
P_{m}(z, s)=\varphi_{m}(z, s)+\sum_{n \equiv 1(24)} c\left(n^{\prime}, y, s\right) e\left(\frac{n}{24} x\right) .
$$

Using (3.4) we find that

$$
\begin{aligned}
c\left(n^{\prime}, y, s\right)= & \sqrt{i} \sum_{c>0} \frac{K\left(m^{\prime}, n^{\prime} ; c\right)}{c^{k}} \\
& \times \int_{-\infty}^{\infty} z^{-k}\left(\frac{4 \pi|m| y}{24 c^{2}|z|^{2}}\right)^{-\frac{k}{2}} M_{\operatorname{sgn}(m) \frac{k}{2}, s-\frac{1}{2}}\left(\frac{4 \pi|m| y}{24 c^{2}|z|^{2}}\right) e\left(\frac{-m x}{24 c^{2}|z|^{2}}-\frac{n x}{24}\right) d x .
\end{aligned}
$$

The integral in (3.10) can be written as

$$
\begin{aligned}
& \left(4 \pi|m| \frac{y}{24}\right)^{-\frac{k}{2}} c^{k} i^{-k} \\
& \times \int_{-\infty}^{\infty}\left(\frac{y-i x}{y+i x}\right)^{-\frac{k}{2}} M_{\operatorname{sgn}(m) \frac{k}{2}, s-\frac{1}{2}}\left(\frac{4 \pi|m| y}{24 c^{2}\left(x^{2}+y^{2}\right)}\right) e\left(\frac{-m x}{24 c^{2}\left(x^{2}+y^{2}\right)}-\frac{n x}{24}\right) d x \\
& =:\left(4 \pi|m| \frac{y}{24}\right)^{-\frac{k}{2}} c^{k} i^{-k} I .
\end{aligned}
$$

The integral $I$ is computed in ([7], p. 33) in the case $m<0$, and the case $m>0$ is similar. Write $x=-y u$. Then

$$
I=y \int_{-\infty}^{\infty}\left(\frac{1-i u}{1+i u}\right)^{\frac{k}{2}} M_{\frac{k}{2}, s-\frac{1}{2}}\left(\frac{4 \pi m}{24 c^{2} y\left(u^{2}+1\right)}\right) e\left(\frac{m u}{24 c^{2} y\left(u^{2}+1\right)}+\frac{n y u}{24}\right) d u .
$$


Now let $A=\frac{n y}{24}$ and $B=\frac{m}{24 c^{2} y}$. By ([18], p. 357) we have

$$
\begin{aligned}
I & =y \Gamma(2 s) \cdot\left\{\begin{array}{l}
\frac{2 \pi \sqrt{|B / A|}}{\Gamma\left(s+\frac{k}{2}\right)} W_{\frac{k}{2}, s-\frac{1}{2}}(4 \pi|A|) J_{2 s-1}(4 \pi \sqrt{|A B|}) \quad \text { if } A>0, \\
\frac{2 \pi \sqrt{|B / A|}}{\Gamma\left(s-\frac{k}{2}\right)} W_{-\frac{k}{2}, s-\frac{1}{2}}(4 \pi|A|) I_{2 s-1}(4 \pi \sqrt{|A B|}) \text { if } A<0,
\end{array}\right. \\
& =\frac{2 \pi \Gamma(2 s) \sqrt{|m / n|}}{c \Gamma\left(s+\operatorname{sgn}(n) \frac{k}{2}\right)} W_{\operatorname{sgn}(n) \frac{k}{2}, s-\frac{1}{2}}\left(4 \pi|n| \frac{y}{24}\right) \cdot\left\{\begin{array}{l}
J_{2 s-1}\left(\frac{\pi}{6 c} \sqrt{|m n|}\right) \text { if } n>0, \\
I_{2 s-1}\left(\frac{\pi}{6 c} \sqrt{|m n|}\right) \text { if } n<0 .
\end{array}\right.
\end{aligned}
$$

Combining this with the expression for $m<0$ from ([7], p. 33), we obtain

$$
I=\frac{2 \pi \Gamma(2 s) \sqrt{|m / n|}}{c \Gamma\left(s+\operatorname{sgn}(n) \frac{k}{2}\right)} W_{\operatorname{sgn}(n) \frac{k}{2}, s-\frac{1}{2}}\left(4 \pi|n| \frac{y}{24}\right) \cdot\left\{\begin{array}{l}
J_{2 s-1}\left(\frac{\pi}{6 c} \sqrt{|m n|}\right) \text { if } m n>0 \\
I_{2 s-1}\left(\frac{\pi}{6 c} \sqrt{|m n|}\right) \text { if } m n<0 .
\end{array}\right.
$$

Using this with (3.10) and (3.11) we find that

$$
\begin{aligned}
c\left(n^{\prime}, y, s\right)= & \frac{2 \pi i^{-k+\frac{1}{2}} \Gamma(2 s) \sqrt{|m / n|}}{\Gamma\left(s+\operatorname{sgn}(n) \frac{k}{2}\right)}\left(4 \pi|m| \frac{y}{24}\right)^{-\frac{k}{2}} W_{\operatorname{sgn}(n) \frac{k}{2}, s-\frac{1}{2}}\left(4 \pi|n| \frac{y}{24}\right) \\
& \times \sum_{c>0} \frac{K\left(m^{\prime}, n^{\prime} ; c\right)}{c} \cdot\left\{\begin{array}{l}
J_{2 s-1}\left(\frac{\pi}{6 c} \sqrt{|m n|}\right) \text { if } m n>0, \\
I_{2 s-1}\left(\frac{\pi}{6 c} \sqrt{|m n|}\right) \text { if } m n<0 .
\end{array}\right.
\end{aligned}
$$

\subsection{Derivatives of nonholomorphic Maass-Poincaré series in weight $\frac{5}{2}$.}

We specialize Proposition 8 to the situation $k=\frac{5}{2}$ and $s=\frac{k}{2}=\frac{5}{4}$. Using (3.7) and (3.8) and noting that $g_{m, n}(s)=0$ for $n<0$, we obtain

$$
P_{m}\left(z, \frac{5}{4}\right)=q^{\frac{m}{24}}-2 \pi \sum_{0<n \equiv 1(24)}\left|\frac{n}{m}\right|^{\frac{3}{4}} L_{m, n}\left(\frac{5}{4}\right) q^{\frac{n}{24}} .
$$

Since $S_{\frac{5}{2}}(\varepsilon)=\{0\}$, we see that

$$
P_{m}\left(z, \frac{5}{4}\right)=0 \quad \text { for } m>0 \text {. }
$$

In order to construct nontrivial forms when $m>0$, we apply the method of [16] and consider the derivative

$$
Q_{m}(z):=\left.\partial_{s}\right|_{s=\frac{5}{4}} P_{m}(z, s)
$$

By (3.6) we have

$$
\Delta_{\frac{5}{2}} \partial_{s} P_{m}(z, s)=(1-2 s) P_{m}(z, s) .
$$

By (3.13) we find that $\Delta_{\frac{5}{2}} Q_{m}(z)=0$; it follows that

$$
Q_{m}(z) \in H_{\frac{5}{2}}^{!}(\varepsilon)
$$

The following proposition gives the Fourier expansion of $Q_{m}(z)$, and is an analogue of Proposition 4 of [16]. 
Proposition 9. For $m>0$ let $Q_{m}(z)$ be defined as in (3.14). Then we have

$$
\begin{aligned}
Q_{m}(z)= & \left.\left(4 \pi m \frac{y}{24}\right)^{-\frac{5}{4}} \partial_{s}\right|_{s=\frac{5}{4}}\left[M_{\frac{5}{4}, s-\frac{1}{2}}\left(4 \pi m \frac{y}{24}\right)-W_{\frac{5}{4}, s-\frac{1}{2}}\left(4 \pi m \frac{y}{24}\right)\right] e\left(\frac{m x}{24}\right) \\
& -\frac{\Gamma^{\prime}}{\Gamma}\left(\frac{5}{2}\right) q^{\frac{m}{24}}-2 \pi \sum_{0<n \equiv 1 \bmod 24}\left(\frac{n}{m}\right)^{\frac{3}{4}} \mathcal{L}_{m, n} \cdot q^{\frac{n}{24}} \\
& -2 \pi \sum_{0>n \equiv 1 \bmod 24}\left|\frac{n}{m}\right|^{\frac{3}{4}} \Gamma\left(\frac{5}{2}\right) L_{m, n}\left(\frac{5}{4}\right) \Gamma\left(-\frac{3}{2}, 4 \pi|n| \frac{y}{24}\right) q^{\frac{n}{24}},
\end{aligned}
$$

where

$$
\mathcal{L}_{m, n}:=\left.\partial_{s}\right|_{s=\frac{5}{4}}\left[L_{m, n}(s)\right]=\left.\sum_{c>0} \frac{K\left(m^{\prime}, n^{\prime}, c\right)}{c} \partial_{s}\right|_{s=\frac{5}{4}}\left[J_{2 s-1}\left(\frac{\pi \sqrt{m n}}{6 c}\right)\right] .
$$

Proof. We compute

$$
\begin{aligned}
\left.\partial_{s}\right|_{s=\frac{5}{4}} P_{m}(z, s)= & \left.\partial_{s}\right|_{s=\frac{5}{4}}\left[\varphi_{m}(z, s)\right] \\
& +\left.\sum_{n \neq 0} \partial_{s}\right|_{s=\frac{5}{4}}\left[g_{m, n}(s) L_{m, n}(s)\right] \cdot\left(4 \pi m \frac{y}{24}\right)^{-\frac{5}{4}} W_{\operatorname{sgn}(n) \frac{5}{4}, \frac{3}{4}}\left(4 \pi|n| \frac{y}{24}\right) e\left(\frac{n x}{24}\right) \\
& +\left.\sum_{n \neq 0} g_{m, n}\left(\frac{5}{4}\right) L_{m, n}\left(\frac{5}{4}\right)\left(4 \pi m \frac{y}{24}\right)^{-\frac{5}{4}} \partial_{s}\right|_{s=\frac{5}{4}}\left[W_{\operatorname{sgn}(n) \frac{5}{4}, s-\frac{1}{2}}\left(4 \pi|n| \frac{y}{24}\right)\right] e\left(\frac{n x}{24}\right) .
\end{aligned}
$$

By (3.12) and (3.13) we see that if $m, n>0$, then

$$
L_{m, n}\left(\frac{5}{4}\right)= \begin{cases}0 & \text { if } m \neq n \\ \frac{1}{2 \pi} & \text { if } m=n .\end{cases}
$$

Therefore the second sum reduces to

$$
-\left.\left(4 \pi m \frac{y}{24}\right)^{-\frac{5}{4}} \partial_{s}\right|_{s=\frac{5}{4}}\left[W_{\operatorname{sgn}(n) \frac{5}{4}, s-\frac{1}{2}}\left(4 \pi m \frac{y}{24}\right)\right] e\left(\frac{m x}{24}\right) .
$$

If $n>0$, then by (3.8), the $n$-th term in the first sum is

$$
\left.\partial_{s}\right|_{s=\frac{5}{4}}\left[g_{m, n}(s) L_{m, n}(s)\right]\left(\frac{m}{n}\right)^{-\frac{5}{4}} q^{\frac{n}{24}} .
$$

Using (3.15) we find that

$$
\left.\partial_{s}\right|_{s=\frac{5}{4}}\left[g_{m, n}(s) L_{m, n}(s)\right]=-\frac{\Gamma^{\prime}}{\Gamma}\left(\frac{5}{2}\right) \delta_{m, n}-2 \pi\left|\frac{m}{n}\right|^{\frac{1}{2}} \mathcal{L}_{m, n}
$$

(where $\delta_{m, n}$ denotes the Kronecker delta function).

If $n<0$, then $g_{m, n}\left(\frac{5}{4}\right)=0$, so the $n$-th term in the first sum is

$$
\begin{aligned}
& -2 \pi\left|\frac{m}{n}\right|^{\frac{1}{2}} \Gamma\left(\frac{5}{2}\right) L_{m, n}\left(\frac{5}{4}\right)\left(4 \pi m \frac{y}{24}\right)^{-\frac{5}{4}} W_{-\frac{5}{4}, \frac{3}{4}}\left(4 \pi|n| \frac{y}{24}\right) e\left(\frac{n x}{24}\right) \\
= & -2 \pi\left|\frac{n}{m}\right|^{\frac{3}{4}} \Gamma\left(\frac{5}{2}\right) L_{m, n}\left(\frac{5}{4}\right) \Gamma\left(-\frac{3}{2}, 4 \pi|n| \frac{y}{24}\right) q^{\frac{n}{24}} .
\end{aligned}
$$

We must evaluate the first two terms which appear in the expansion of $Q_{m}(z)$.

Lemma 10. Suppose that $k \notin \mathbb{Z}$ and that $m>0$. Then

$$
\begin{gathered}
\left.\left(4 \pi m \frac{y}{24}\right)^{-\frac{k}{2}} \partial_{s}\right|_{s=\frac{k}{2}}\left[M_{\frac{k}{2}, s-\frac{1}{2}}\left(4 \pi m \frac{y}{24}\right)-W_{\frac{k}{2}, s-\frac{1}{2}}\left(4 \pi m \frac{y}{24}\right)\right] e\left(\frac{m x}{24}\right)-\frac{\Gamma^{\prime}}{\Gamma}(k) q^{\frac{m}{24}} \\
=\left(\pi \cot \pi k-(-1)^{k} \pi \csc \pi k\right) q^{\frac{m}{24}}+(-1)^{k} \Gamma(k) \Gamma\left(1-k,-4 \pi m \frac{y}{24}\right) q^{\frac{m}{24}} .
\end{gathered}
$$


Proof. By (13.14.33) of [21], we have

$$
\begin{aligned}
& \left.\partial_{s}\right|_{s=\frac{k}{2}}\left[M_{\frac{k}{2}, s-\frac{1}{2}}(y)-W_{\frac{k}{2}, s-\frac{1}{2}}(y)\right] \\
& =\left.\partial_{s}\right|_{s=\frac{k}{2}}\left[M_{\frac{k}{2}, s-\frac{1}{2}}(y)-\frac{\Gamma(1-2 s)}{\Gamma(1-s-k / 2)} M_{\frac{k}{2}, s-\frac{1}{2}}(y)-\frac{\Gamma(2 s-1)}{\Gamma(s-k / 2)} M_{\frac{k}{2}, \frac{1}{2}-s}(y)\right] \\
& =\left.\partial_{s}\right|_{s=\frac{k}{2}}\left[1-\frac{\Gamma(1-2 s)}{\Gamma(1-s-k / 2)}\right] M_{\frac{k}{2}, \frac{k-1}{2}}(y)-\left.\partial_{s}\right|_{s=\frac{k}{2}}\left[\frac{\Gamma(2 s-1)}{\Gamma(s-k / 2)}\right] M_{\frac{k}{2}, \frac{1-k}{2}}(y) \\
& =\frac{\Gamma^{\prime}}{\Gamma}(1-k) M_{\frac{k}{2}, \frac{k-1}{2}}(y)-\Gamma(k-1) M_{\frac{k}{2}, \frac{1-k}{2}}(y) .
\end{aligned}
$$

For the first term, we have (by (3.7))

$$
y^{-\frac{k}{2}} M_{\frac{k}{2}, \frac{k-1}{2}}(y)=e^{-\frac{y}{2}} .
$$

For the second term, we use (13.14.2) and (13.6.5) of [21] to obtain

$$
y^{-\frac{k}{2}} M_{\frac{k}{2}, \frac{1-k}{2}}(y)=(-1)^{k-1}(1-k) e^{-\frac{y}{2}}(\Gamma(1-k)-\Gamma(1-k,-y)) .
$$

Therefore, the quantity on the left in (3.16) reduces to

$$
\left(\frac{\Gamma^{\prime}}{\Gamma}(1-k)-(-1)^{k} \Gamma(k) \Gamma(1-k)-\frac{\Gamma^{\prime}}{\Gamma}(k)\right) q^{\frac{m}{24}}+(-1)^{k} \Gamma(k) \Gamma\left(1-k,-4 \pi m \frac{y}{24}\right) q^{\frac{m}{24}} .
$$

The lemma follows after using the reflection formula

$$
\Gamma(z) \Gamma(1-z)=\pi \csc \pi z,
$$

and its logarithmic derivative

$$
\frac{\Gamma^{\prime}}{\Gamma}(1-z)-\frac{\Gamma^{\prime}}{\Gamma}(z)=\pi \cot \pi z
$$

\subsection{Proof of Theorem 1}

The next Proposition, which follows directly from Proposition 9 and Lemma 10, summarizes the two constructions.

Proposition 11. Let $P_{m}\left(z, \frac{5}{4}\right)$ and $Q_{m}(z)$ be as defined in (3.12) and (3.14). For negative $m \equiv 1(\bmod 24)$, define

$$
h_{m}(z):=P_{m}\left(z, \frac{5}{4}\right)=q^{\frac{m}{24}}-2 \pi \sum_{0<n \equiv 1(24)}\left|\frac{n}{m}\right|^{\frac{3}{4}} L_{m, n}\left(\frac{5}{4}\right) q^{\frac{n}{24}},
$$

and for positive $m \equiv 1(\bmod 24)$, define

$$
\begin{aligned}
h_{m}(z):=\frac{1}{\Gamma\left(\frac{5}{2}\right)} Q_{m}(z)= & -\frac{4}{3} i \sqrt{\pi} q^{\frac{m}{24}}-\frac{8}{3} \sqrt{\pi} \sum_{0<n \equiv 1(24)}\left(\frac{n}{m}\right)^{\frac{3}{4}} \mathcal{L}_{m, n} q^{\frac{n}{24}} \\
& +i \beta(-m y) q^{\frac{m}{24}}-2 \pi \sum_{0>n \equiv 1(24)}\left|\frac{n}{m}\right|^{\frac{3}{4}} L_{m, n}\left(\frac{5}{4}\right) \beta(|n| y) q^{\frac{n}{24}} .
\end{aligned}
$$

Then $h_{m} \in H_{\frac{5}{2}}^{!}(\varepsilon)$, and for negative $m$ we have $h_{m} \in M_{\frac{5}{2}}^{!}(\varepsilon)$. 
Theorem 1 now follows quickly.

Proof of Theorem 1. By the discussion following Lemma 7, there can be at most one element of $H_{\frac{5}{2}}^{!}(\varepsilon)$ having a Fourier expansion of the form (3.17) and at most one having an expansion of the form (3.18). By the same discussion we find that $\left\{h_{m}\right\}$ spans $f \in H_{\frac{5}{2}}^{!}(\varepsilon)$. For $m>0$ we have

$$
\xi_{\frac{5}{2}} h_{m}(z)=\left(\frac{6}{\pi m}\right)^{\frac{3}{2}} q^{-\frac{m}{24}}+2 \pi \sum_{0>n \equiv 1(24)}\left|\frac{n}{m}\right|^{\frac{3}{4}} L_{m, n}\left(\frac{5}{4}\right)\left(\frac{6}{\pi|n|}\right)^{\frac{3}{2}} q^{-\frac{n}{24}} .
$$

By comparing principal parts, we conclude that $\xi_{\frac{5}{2}} h_{m}=\left(\frac{6}{\pi m}\right)^{\frac{3}{2}} g_{m}$.

We have

$$
\overline{K(m, n ; c)}=K(m, n ; c),
$$

which results from a computation using the relation $s(-d, c)=-s(d, c)$. It follows from (3.18) that the coefficients $p_{m}^{+}(n)$ are real for $m \neq n$, and that $p_{m}^{+}(m)$ is not real for $m>0$ (cf. (1.3)).

\section{Rademacher's formula for $p(n)$}

Writing $n=24 n^{\prime}-1$, we obtain

$$
\xi_{k}\left(h_{1}(z)\right)=\left(\frac{6}{\pi}\right)^{\frac{3}{2}} q^{-\frac{1}{24}}+\sum_{n^{\prime}>0} 2 \pi n^{-\frac{3}{4}}\left(\frac{6}{\pi}\right)^{\frac{3}{2}} \sum_{c>0} \frac{K\left(0,-n^{\prime} ; c\right)}{c} I_{\frac{3}{2}}\left(\frac{\pi \sqrt{24 n^{\prime}-1}}{6 c}\right) q^{n^{\prime}-\frac{1}{24}} .
$$

The $I$-Bessel function satisfies

$$
I_{\frac{3}{2}}(x)=\sqrt{\frac{2}{\pi}}\left(\frac{x \cosh x-\sinh x}{x^{3 / 2}}\right)=\sqrt{\frac{2}{\pi}} x^{1 / 2} \frac{d}{d x}\left(\frac{\sinh x}{x}\right),
$$

so that

$$
I_{\frac{3}{2}}\left(\frac{\pi}{6} \sqrt{\frac{2}{3}} \sqrt{n-\frac{1}{24}}\right)=\frac{1}{2 \sqrt{2}} \frac{c^{\frac{3}{2}}}{\pi^{2}}(24 n-1)^{\frac{3}{4}} \frac{d}{d n}\left(\frac{\sinh \left(\frac{\pi}{c} \sqrt{\frac{2}{3}} \sqrt{n-\frac{1}{24}}\right)}{\sqrt{n-\frac{1}{24}}}\right) .
$$

Together with Theorem 1 this gives

$$
\begin{aligned}
\eta^{-1}(z) & =\left(\frac{\pi}{6}\right)^{\frac{3}{2}} \xi_{k}\left(h_{1}(z)\right) \\
& =q^{-1 / 24}+\frac{1}{\pi \sqrt{2}} \sum_{n>0} \sum_{c>0} K(0,-n ; c) \sqrt{c} \frac{d}{d n}\left(\frac{\sinh \left(\frac{\pi}{c} \sqrt{\frac{2}{3}} \sqrt{n-\frac{1}{24}}\right)}{\sqrt{n-\frac{1}{24}}}\right) q^{n-\frac{1}{24}} .
\end{aligned}
$$

Equating coefficients of $q^{n-\frac{1}{24}}$ gives Rademacher's exact formula

$$
p(n)=\frac{1}{\pi \sqrt{2}} \sum_{c>0} K(0,-n ; c) \sqrt{c} \frac{d}{d n}\left(\frac{\sinh \left(\frac{\pi}{c} \sqrt{\frac{2}{3}} \sqrt{n-\frac{1}{24}}\right)}{\sqrt{n-\frac{1}{24}}}\right) .
$$

\section{Proof of Theorem 3}

We proceed as in [16] and [15]. Let $\mathcal{F}(Y)$ denote the usual fundamental domain for $\mathrm{SL}_{2}(\mathbb{Z})$, truncated at height $Y$. We have the following (see, for example, [4], Section 9). 
Lemma 12. Suppose that $k \in \frac{1}{2} \mathbb{Z}$, that $h \in H_{2-k}^{!}(\varepsilon)$, and that $g \in M_{k}^{!}(\bar{\varepsilon})$. Then we have

$$
\int_{\mathcal{F}(Y)} g(z) \overline{\xi_{2-k} h(z)} \cdot y^{k} \frac{d x d y}{y^{2}}=\int_{-\frac{1}{2}+i Y}^{\frac{1}{2}+i Y} g(z) h(z) d z .
$$

Proof. We have

$$
g(z) \overline{\xi_{2-k} h(z)} \cdot y^{k} \frac{d x d y}{y^{2}}=g(z) \frac{\partial h}{\partial \bar{z}} d z \wedge d \bar{z}=-d(g(z) h(z) d z) .
$$

The result follows from Stokes' theorem.

For positive $n \equiv 1(\bmod 24)$ we write the forms $g_{n}$ from $(1.2)$ as

$$
g_{n}(z)=q^{-\frac{n}{24}}+\sum_{0<j \equiv 23(24)} c_{n}(j) q^{\frac{j}{24}} .
$$

To prove Theorem 3, we compute

$$
\begin{aligned}
& \int_{-\frac{1}{2}+i Y}^{\frac{1}{2}+i Y} g_{n}(z) h_{m}(z) d z \\
& =\int_{-\frac{1}{2}+i Y}^{\frac{1}{2}+i Y}\left(q^{-\frac{n}{24}}+\sum_{0<j \equiv 23(24)} c_{n}(j) q^{\frac{j}{24}}\right)\left(-\frac{4}{3} i \sqrt{\pi} q^{\frac{m}{24}}-\frac{8 \sqrt{\pi}}{3} \sum_{0<k \equiv 1(24)}\left(\frac{k}{m}\right)^{\frac{3}{4}} \mathcal{L}_{m, k} q^{\frac{k}{24}}\right. \\
& \left.+i \beta(-m y) q^{\frac{m}{24}}-\underset{0>k \equiv 1(24)}{2 \pi}\left|\frac{k}{m}\right|^{\frac{3}{4}} L_{m, k}\left(\frac{5}{4}\right) \beta(|k| y) q^{\frac{k}{24}}\right) d z
\end{aligned}
$$

By Lemma 3.4 of [8] we have (for some constant $C$ )

$$
\left|c_{n}(j)\right| \ll e^{C \sqrt{j}}, \quad\left|\mathcal{L}_{m, k}\right| \ll e^{C \sqrt{k}}, \quad \text { and } \quad\left|L_{m, k}\left(\frac{5}{4}\right)\right| \ll e^{C \sqrt{|k|}}
$$

From ([21], (8.11.2)) we have

$$
\beta(|k| Y)=\Gamma\left(-\frac{3}{2}, \frac{\pi|k| Y}{6}\right) \sim\left(\frac{\pi|k| Y}{6}\right)^{-\frac{5}{2}} e^{-\frac{\pi|k| Y}{6}} \quad \text { as } \quad|k| Y \rightarrow \infty .
$$

From these estimates we conclude that the expression in (5.1) can be integrated term by term. Also note that for each pair $j, k$ with $j \equiv 23(\bmod 24), k \equiv 1(\bmod 24)$, and $j \neq-k$, we have

$$
\int_{-\frac{1}{2}+i Y}^{\frac{1}{2}+i Y} q^{\frac{j}{24}} q^{\frac{k}{24}} d z=0
$$

Since $n \neq m$ we obtain

$$
\begin{aligned}
& \int_{-\frac{1}{2}+i Y}^{\frac{1}{2}+i Y} g_{n}(z) h_{m}(z) d z \\
&=-\frac{8 \sqrt{\pi}}{3}\left(\frac{n}{m}\right)^{\frac{3}{4}} \mathcal{L}_{m, n}-2 \pi \sum_{0>k \equiv 1(24)} c_{n}(|k|)\left|\frac{k}{m}\right|^{\frac{3}{4}} L_{m, k}\left(\frac{5}{4}\right) \beta(|k| Y) \\
&=p_{m}^{+}(n)-2 \pi \sum_{0>k \equiv 1(24)} c_{n}(|k|)\left|\frac{k}{m}\right|^{\frac{3}{4}} L_{m, k}\left(\frac{5}{4}\right) \beta(|k| Y) .
\end{aligned}
$$


By (5.2) and (5.3) we see that the sum goes to zero as $Y \rightarrow \infty$. So by Lemma 12, (1.4), and Theorem 1 we obtain

$$
\left(\frac{6}{\pi m}\right)^{\frac{3}{2}}\left\langle g_{n}, g_{m}\right\rangle_{\mathrm{reg}}=p_{m}^{+}(n) .
$$

\section{Proof of Theorem 5}

Let $\ell \geq 5$ be prime. On the spaces $M_{-\frac{1}{2}}^{!}(\bar{\varepsilon})$ and $H_{\frac{5}{2}}^{!}(\varepsilon)$ we have the Hecke operators $T_{-\frac{1}{2}}\left(\ell^{2}\right)$ and $T_{\frac{5}{2}}\left(\ell^{2}\right)$. For

$$
f=\sum_{n \equiv 23(24)} a(n) q^{n / 24} \in M_{-\frac{1}{2}}^{!}(\bar{\varepsilon})
$$

we have

$$
f \mid T_{-\frac{1}{2}}\left(\ell^{2}\right)=\sum_{n \equiv 23(24)}\left(a\left(\ell^{2} n\right)+\ell^{-2}\left(\frac{-3 n}{\ell}\right) a(n)+\ell^{-3} a\left(n / \ell^{2}\right)\right) q^{n / 24} .
$$

There are also Hecke operators on the space $H_{\frac{5}{2}}^{!}(\varepsilon)$ (see, e.g. [13] or ([12], Section 7)). For primes $\ell \geq 5$, our Hecke operator $T_{\frac{5}{2}}\left(\ell^{2}\right)$ is the same as the usual Hecke operator of index $\ell^{2}$ on the space $H_{\frac{5}{2}}^{!}\left(\Gamma_{0}(576),\left(\frac{12}{\bullet}\right)\right)$, conjugated by $\left(\begin{array}{cc}24 & 0 \\ 0 & 1\end{array}\right)$. In particular, for

$$
g=\sum_{n \equiv 1(24)} b(n) q^{n / 24} \in M_{\frac{5}{2}}^{!}(\varepsilon)
$$

we have

$$
g \mid T_{\frac{5}{2}}\left(\ell^{2}\right)=\sum_{n \equiv 1(24)}\left(b\left(\ell^{2} n\right)+\ell\left(\frac{3 n}{\ell}\right) b(n)+\ell^{3} b\left(n / \ell^{2}\right)\right) q^{n / 24},
$$

and the action of $T_{\frac{5}{2}}\left(\ell^{2}\right)$ on the holomorphic part of a form $h \in H_{\frac{5}{2}}^{!}(\varepsilon)$ is given by the same formula. For $h \in H_{\frac{5}{2}}^{!}(\varepsilon)$ we have the relation

$$
\xi_{\frac{5}{2}}\left(h \mid T_{\frac{5}{2}}\left(\ell^{2}\right)\right)=\ell^{3}\left(\xi_{\frac{5}{2}} h\right) \mid T_{-\frac{1}{2}}\left(\ell^{2}\right)
$$

(see [12], Proposition 7).

For $m>0$, we have $g_{m}=q^{-m / 24}+O\left(q^{1 / 24}\right)$. Since the $g_{m}$ form a basis for $M_{-\frac{1}{2}}^{!}(\bar{\varepsilon})$, comparing principal parts gives the formula

$$
g_{m} \mid T_{-\frac{1}{2}}\left(\ell^{2}\right)=\ell^{-3} g_{\ell^{2} m}+\left(\frac{3 m}{\ell}\right) \ell^{-2} g_{m} .
$$

For $m<0$ we find in a similar way that

$$
h_{m} \mid T_{\frac{5}{2}}\left(\ell^{2}\right)=\ell^{3} h_{\ell^{2} m}+\left(\frac{3 m}{\ell}\right) \ell h_{m},
$$

which gives the theorem in this case.

Suppose that $m>0$. By Theorem 1 we have $h_{m} \mid T_{\frac{5}{2}}\left(\ell^{2}\right)=\sum c_{n} h_{n}$ for some coefficients $c_{n}$, from which we obtain

$$
\xi_{\frac{5}{2}}\left(h_{m} \mid T_{\frac{5}{2}}\left(\ell^{2}\right)\right)=\sum \overline{c_{n}}\left(\frac{6}{\pi n}\right)^{\frac{3}{2}} g_{n} .
$$


On the other hand, the relation (6.1) together with (6.2) gives

$$
\xi_{\frac{5}{2}}\left(h_{m} \mid T_{\frac{5}{2}}\left(\ell^{2}\right)\right)=\left(\frac{6}{\pi m}\right)^{\frac{3}{2}}\left(g_{\ell^{2} m}+\left(\frac{3 m}{\ell}\right) \ell g_{m}\right) \text {. }
$$

Since the $\left\{g_{m}\right\}$ are linearly independent, we see that the only nonzero coefficients in this linear combination are $c_{\ell^{2} m}=\ell^{3}$ and $c_{m}=\left(\frac{3 m}{\ell}\right) \ell$. The theorem follows.

\section{Competing interests}

The authors declare that they have no competing interests.

\section{Acknowledgements}

We thank Kathrin Bringmann, Jan Bruinier, and Karl Mahlburg for their comments, and we thank the referee for suggestions which improved our exposition.

The first author was supported by a grant from the Simons Foundation (\#208525 to Scott Ahlgren).

Received: 12 March 2015 Accepted: 3 June 2015

Published online: 02 December 2015

\section{References}

1. Ahlgren, S: Hecke relations for traces of singular moduli. Bull. Lond. Math. Soc. 44(1), 99-105 (2012). doi:10.1112/blms/bdr072

2. Ahlgren, S, Kim, B: Mock modular grids and Hecke relations for mock modular forms. Forum Math. 26(4), 1261-1287 (2014). doi:10.1515/forum-2012-0011

3. Andersen, N: Singular invariants and coefficients of weak harmonic Maass forms of weight 5/2. Preprint. http://arxiv.org/abs/1410.7349. 1410.7349

4. Borcherds, R: Automorphic forms with singularities on Grassmannians. Invent. Math. 132(3), 491-562 (1998). doi:10.1007/s002220050232

5. Bringmann, K, Diamantis, N, Raum, M: Mock period functions, sesquiharmonic Maass forms, and non-critical values of $L$-functions. Adv. Math. 233, 115-134 (2013). doi:10.1016/j.aim.2012.09.025

6. Bringmann, K, Ono, K: The $f(q)$ mock theta function conjecture and partition ranks. Invent. Math. 165(2), 243-266 (2006). doi:10.1007/s00222-005-0493-5

7. Bruinier, $\mathrm{JH}$ : Borcherds Products on $\mathrm{O}(2, l)$ and Chern Classes of Heegner divisors. Lecture Notes in Mathematics, Vol. 1780. Springer, Berlin (2002). doi:10.1007/b83278. http://dx.doi.org/10.1007/b83278

8. Bruinier, JH, Funke, J: On two geometric theta lifts. Duke Math. J. 125(1), 45-90 (2004). doi:10.1215/S0012-7094-04-12513-8

9. Bruinier, JH, Funke, J, Imamoḡlu, Ö: Regularlized theta liftings and periods of modular functions. J. Reine Angew. Math. 703, 43-93 (2015). doi:10.1515/crelle-2013-0035

10. Bruinier, JH, Jenkins, P, Ono, K: Hilbert class polynomials and traces of singular moduli. Math. Ann. 334(2), 373-393 (2006). doi:10.1007/s00208-005-0723-6

11. Bruinier, JH, Ono, K: Algebraic formulas for the coefficients of half-integral weight harmonic weak Maass forms. Adv. Math. 246, 198-219 (2013). doi:10.1016/j.aim.2013.05.028

12. Bruinier, JH, Ono, K: Heegner divisors, $L$-functions and harmonic weak Maass forms. Ann. Math. (2). 172(3), 2135-2181 (2010). doi:10.4007/annals.2010.172.2135

13. Bruinier, JH, Stein, $\mathrm{O}$ : The Weil representation and Hecke operators for vector valued modular forms. Math. Z 264(2), 249-270 (2010). doi:10.1007/s00209-008-0460-0

14. Duke, W, Imamoḡlu, Ö, Tóth, Á: Cycle integrals of the $j$-function and mock modular forms. Ann. of Math. 173(2), 947-981 (2011). doi:10.4007/annals.2011.173.2.8

15. Duke, W, Imamoḡlu, Ö, Tóth, Á: Real quadratic analogs of traces of singular moduli. Int. Math. Res. Not. 13, 3082-3094 (2011) doi:10.1093/imrn/rnq159

16. Duke, W, Imamoḡlu, Ö, Tóth, Á: Regularized inner products of modular functions. Ramanujan J. to appear. doi:10.1007/s11139-013-9544-5

17. Guerzhoy, P: On weak harmonic Maass-modular grids of even integral weights. Math. Res. Lett. 16(1), 59-65 (2009). doi:10.4310/MRL.2009.v16.n1.a7

18. Hejhal, D: The Selberg Trace Formula for PSL(2, R), Vol. 2. Lecture Notes in Mathematics, Vol. 1001. Springer, Berlin (1983)

19. Iwaniec, H: Topics in Classical Automorphic Forms. Graduate Studies in Mathematics, Vol. 17. American Mathematical Society, Providence, RI (1997)

20. Jeon, D, Kang, S-Y, Kim, CH: Weak Maass-Poincaré series and weight 3/2 mock modular forms. J. Number Theory. 133(8), 2567-2587 (2013). doi:10.1016/j.jnt.2013.01.011

21. NIST Digital Library of Mathematical Functions. Release 1.0.9 of 2014-08-29. http://dlmf.nist.gov/

22. Ono, K: Unearthing the visions of a master: harmonic Maass forms and number theory. In: Current Developments in Mathematics, 2008, pp. 347-454. Int. Press, Somerville, MA, (2009)

23. Rademacher, $\mathrm{H}$ : On the partition function $p(n)$. Proc. London Math. Soc. 43(4), 241-254 (1937). doi:10.1112/plms/s2-43.4.241

24. Zagier, D: Ramanujan's mock theta functions and their applications (d'après Zwegers and Bringmann-Ono). Astérisque. 326, 143-164 (2010). Exp. No. 986, vii-viii, Séminaire Bourbaki. Vol. 2007/2008

25. Zagier, D: Traces of singular moduli. In: Motives, Polylogarithms and Hodge Theory, Part I (Irvine, CA, 1998), Int. Press Lect. Ser., pp. 211-244. Int. Press, Somerville, MA, (2002) 